\title{
Application of Social Cognitive Theory in Obesity Prevention: A Rapid Review
}

\author{
${ }^{1}$ Chiranjivi Adhikari, ${ }^{1}$ Avash Puri, ${ }^{1}$ Dipty Thapa, ${ }^{1}$ Rebishna Thapa, ${ }^{1}$ Sonam Magar, ${ }^{1}$ Sunil GC \\ ${ }^{1}$ Public Health Program, School of Health \& Allied Sciences, Pokhara University
}

\begin{abstract}
Obesity, as a result of including the chronic positive energy balance, is associated with many chronic diseases. Prevalence of obesity is increasing worldwide, including in selected developing countries, from previous very low status. Association or causality of one or more constructs of social cognitive theory to prevent and control obesity is paramount for program interventions.

We searched titles and abstracts using End Note Software and then approached original articles in databases of PubMed, Google Scholar and Health Inter-Network Access to Research Initiative (HINARI) in English language published between Jan 1, 2000 and Jan 10, 2015. The articles included only when one or more of the constructs of social cognitive theory viz. reciprocal determinism, behavioral capability, observational learning, reinforcements, expectations and self-efficacy were examined quantitatively with obesity or overweight. The data from the articles were then summarized and interpreted.

Out of 90 accessed and reviewed full-text articles, 22 included in the review, mostly of which studies were conducted in developing countries. The review results showed that intervening the constructs of social cognitive theory was found effective in preventing obesities including childhood obesities. In studies where media campaign was added with social cognitive theory, logistic regression results demonstrated that behavior change was greater among women.

Self-efficacy and social support are the important constructs of social cognitive/learning theory to be effective and associated in obesity prevention, others remaining modest. It is suggested that the constructs are integrated with media campaign and ecological components when intervened.
\end{abstract}

Key words: Social, cognitive, obesity, overweight, behaviour, self-efficacy, physical.

Correspondence address: Chiranjivi Adhikari

Public Health Program, School of Health and Allied Sciences,

Faculty of Health Sciences, Pokhara University, Kaski, Nepal

E-mail: chiranadhikari@gmail.com

\section{INTRODUCTION}

Obesity is a burgeoning medical condition in which excess body fat has accumulated to the extent that it may have a negative effect on health, leading to reduced life expectancy and/or increased health problems. ${ }^{1}$ People are considered obese when their body mass index (BMI) a measurement obtained by dividing a person's weight by the square of the person's height, exceeds $30 \mathrm{~kg} / \mathrm{m}^{2}$, with the range $25-30 \mathrm{~kg} / \mathrm{m}^{2}$ defined as overweight. ${ }^{2}$ Obesity is now so common within the world's population that it is beginning to replace under nutrition and infectious diseases as the most significant contributor to ill health.
In particular, obesity is associated with diabetes mellitus, coronary heart disease, certain forms of cancer, and sleep-breathing disorders. Obesity as a measurement only accounts weight and height and neither it takes into account the morbidity and mortality associated with more modest degrees of overweight nor the detrimental effect of intraabdominal fat. The global epidemic of obesity results from a combination of genetic susceptibility, increased availability of high-energy foods and decreased requirement for physical activity in modern society. Obesity should no longer be regarded simply as a cosmetic problem affecting 
certain individuals, but an epidemic that threatens global well-being. ${ }^{2}$ Social Cognitive Theory (SCT) states that learning occurs in a social context with dynamic and reciprocal interaction of the person, environment and behavior. Because of the unique feature of SCT that emphasis on social influence and its emphasis on external and internal social reinforcement - it considers unique way by which an individual acquires and maintains behavior whilst considering the social environment.

\section{METHODS}

\section{Search Strategy and Selection Criteria}

We searched PubMed, Google Scholar and HINARI for published articles from 2000 Jan 1 to 2015 Jan 10 . The articles were searched, retrieved and managed by using EndNote (version X3) software. List of key words entered and outputs are shown in table1.

\section{Table.1: Search strategy and selection criteria}

\begin{tabular}{|c|l|}
\hline S.N & \multicolumn{1}{|c|}{ Search Item (Items found) } \\
\hline 1. & $\begin{array}{l}\text { social marketing, obesity OR overweight } \\
\text { prevention }\{233\}\end{array}$ \\
\hline 2. & $\begin{array}{l}\text { obesity OR overweight prevention, stages of } \\
\text { changes, transtheoritical model }\{9\}\end{array}$ \\
\hline 3. & $\begin{array}{l}\text { Theory of reasoned action, obesity OR } \\
\text { overweight prevention, developing countries }\{5\}\end{array}$ \\
\hline 4. & $\begin{array}{l}\text { obesity/overweight treatment, social cognitive } \\
\text { theory }\{79\}\end{array}$ \\
\hline 5. & $\begin{array}{l}\text { female obesity/overweight prevention, behavioral } \\
\text { learning theory }\{12\}\end{array}$ \\
\hline
\end{tabular}

\section{Screening and Data Extraction}

Only published articles in English language and based on obesity in developing countries as well as some developed countries were reviewed. The data extraction was done manually (fig. 1).

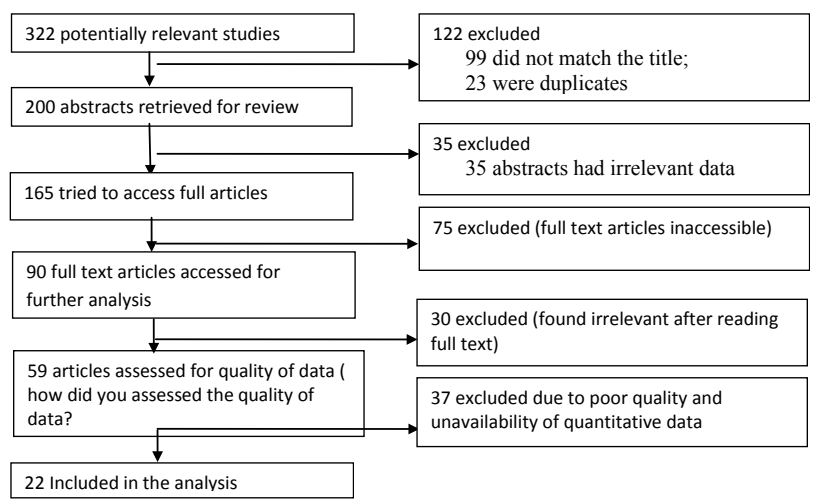

Fig. 1: Screening and data extraction

\section{RESULTS}

Nineteen interventional and three observational studies were reviewed. The sample size ranged from 22 to 15000 . Interventional studies ranged from single group to randomized controlled trials, whereas observational studies included longitudinal and cross-sectional studies. Social cognitive/learning theory goes beyond behaviorism and thus corresponds to opening the black-box of human beings. It thus includes social learning and imitation, self-identity, thinking and rationalization. These constructs have been found mostly effective and associated in most of the studies (table 2).

Table 2: Study design, results and conclusions of 22 studies

\begin{tabular}{|c|c|c|c|c|c|c|}
\hline Study & $\begin{array}{l}\text { Model/ } \\
\text { theory and } \\
\text { constructs }\end{array}$ & $\begin{array}{c}\text { Research/ } \\
\text { Study design }\end{array}$ & $\begin{array}{l}\text { Sample } \\
\text { Size }\end{array}$ & $\begin{array}{c}\text { Results (including statistic } \\
\text { and p-value) }\end{array}$ & Conclusion & Remarks \\
\hline $\begin{array}{l}\text { Gray- } \\
\text { Donald } \\
\text { et al. }{ }^{3}\end{array}$ & $\begin{array}{l}\text { Social } \\
\text { Learning } \\
\text { Theory }\end{array}$ & $\begin{array}{l}\text { Prospective } \\
\text { interventional } \\
\text { study }\end{array}$ & 219 & $\begin{array}{l}\text { This intervention had only } \\
\text { a minor impact on dietary } \\
\text { behavior of the sample. }\end{array}$ & $\begin{array}{l}\text { Finding ways } \\
\text { of encouraging } \\
\text { appropriate } \\
\text { body weight } \\
\text { and activity } \\
\text { levels remains a } \\
\text { challenge. }\end{array}$ & $\begin{array}{l}\text { SCT was not } \\
\text { found to be } \\
\text { Effective. }\end{array}$ \\
\hline $\begin{array}{l}\text { Dewar et } \\
\text { al. }{ }^{4}\end{array}$ & $\begin{array}{l}\text { Social } \\
\text { Cognitive } \\
\text { Theory } \\
\text { model } \\
\text { on, } \\
\text { Adolescent } \\
\text { Girls. }\end{array}$ & Interventional & $\begin{array}{l}235 \\
\text { Subjects }\end{array}$ & $\begin{array}{l}\text { Current findings indicate } \\
\text { a large proportion of the } \\
\text { variance for physical } \\
\text { activity and intention } \\
(28 \% \text { and } 34 \%) \text { remains } \\
\text { unexplained. }\end{array}$ & $\begin{array}{l}\text { The proposed } \\
\text { pathways in } \\
\text { the SCT model } \\
\text { were not fully } \\
\text { supported }\end{array}$ & $\begin{array}{l}\text { SCT was } \\
\text { not found } \\
\text { effective, may } \\
\text { be of low } \\
\text { sample size. }\end{array}$ \\
\hline
\end{tabular}




\begin{tabular}{|c|c|c|c|c|c|c|}
\hline $\begin{array}{l}\text { Dressler } \\
\text { et al. }{ }^{5}\end{array}$ & $\begin{array}{l}\text { Social } \\
\text { Cognitive } \\
\text { theory } \\
\text { on Urban } \\
\text { Middle } \\
\text { Aged } \\
\text { women }\end{array}$ & Interventional & $\begin{array}{l}330 \\
\text { women's }\end{array}$ & $\begin{array}{l}\text { Suggest that personal, } \\
\text { behavioral, and self- } \\
\text { identity factors can help } \\
\text { to explain some weight } \\
\text { variation observed among } \\
\text { women living in similar } \\
\text { obesogenic, low-income } \\
\text { environments. }\end{array}$ & $\begin{array}{l}\text { Results showed } \\
\text { that only self- } \\
\text { efficacy was } \\
\text { associated with } \\
\text { physical activity. }\end{array}$ & $\begin{array}{l}\text { Integration of } \\
\text { other models, } \\
\text { that include } \\
\text { ecological } \\
\text { components, } \\
\text { should be } \\
\text { fostered. }\end{array}$ \\
\hline $\begin{array}{l}\text { Patrick } \\
\text { et al. }{ }^{6}\end{array}$ & $\begin{array}{l}\text { Social } \\
\text { Cognitive } \\
\text { Theory } \\
\text { on Young } \\
\text { Adults }\end{array}$ & $\begin{array}{l}\text { Randomized } \\
\text { Control Trial }\end{array}$ & $\begin{array}{l}404 \\
\text { subjects }\end{array}$ & $\begin{array}{l}\text { Theory-driven, evidence- } \\
\text { based strategies for } \\
\text { physical activity, sedentary } \\
\text { behavior, and dietary intake } \\
\text { can be embedded in an } \\
\text { intervention. }\end{array}$ & $\begin{array}{l}\text { Using social } \\
\text { and mobile } \\
\text { technologies to } \\
\text { promote healthy } \\
\text { weight-related } \\
\text { behaviors in } \\
\text { young adults is } \\
\text { effective. }\end{array}$ & $\begin{array}{l}\text { Mobiles and } \\
\text { internet also } \\
\text { serve as a } \\
\text { effective } \\
\text { interventional } \\
\text { components } \\
\text { in obesity } \\
\text { prevention. }\end{array}$ \\
\hline $\begin{array}{l}\text { Sharpe } \\
\text { et al. }\end{array}$ & $\begin{array}{l}\text { Social } \\
\text { Cognitive } \\
\text { Theory } \\
\text { Targeted } \\
\text { on middle- } \\
\text { aged women }\end{array}$ & $\begin{array}{l}\text { Community- } \\
\text { based } \\
\text { intervention }\end{array}$ & $\begin{array}{l}2768 \\
\text { subjects }\end{array}$ & $\begin{array}{l}\text { Women in the behavioral } \\
\text { intervention had } \\
\text { statistically significant ( } \mathrm{n}= \\
234 \text {, pre } 822 \text {, post) (P-value } \\
<0.001) \text { positive changes. } \\
\text { Media exposed women } \\
\text { had statistically significant } \\
\text { (P-value }<0.001 \text { ) pre- to } \\
\text { post program differences } \\
\text { on knowledge of mapped } \\
\text { routes. }\end{array}$ & $\begin{array}{l}\text { No-intervention } \\
\text { women had } \\
\text { significant pre- } \\
\text { to post program } \\
\text { differences on } \\
\text { physical activity } \\
\text { minutes, walking, } \\
\text { and knowledge of } \\
\text { mapped routes. }\end{array}$ & $\begin{array}{l}\text { Women must } \\
\text { be exposed } \\
\text { to medias } \\
\text { for effective } \\
\text { intervention } \\
\text { implementation } \\
\text { regarding } \\
\text { obesity } \\
\text { prevention and } \\
\text { control. }\end{array}$ \\
\hline $\begin{array}{l}\text { Briley et } \\
\text { al. }{ }^{8}\end{array}$ & $\begin{array}{l}\text { Social } \\
\text { Cognitive } \\
\text { Theory }\end{array}$ & $\begin{array}{l}\text { Multicenter } \\
\text { Randomized } \\
\text { Control Trial }\end{array}$ & $\begin{array}{l}1546 \\
\text { subjects }\end{array}$ & $\begin{array}{l}\text { Findings of this trial } \\
\text { suggest that the lifestyle } \\
\text { mediated improvement } \\
\text { of glycemic control in } \\
\text { obese pregnant women } \\
\text { can minimize the risk of } \\
\text { pregnancy complications. }\end{array}$ & $\begin{array}{l}\text { Obesity is related } \\
\text { with pregnancy } \\
\text { complications as } \\
\text { well. }\end{array}$ & $\begin{array}{l}\text { SCT can be } \\
\text { used as a } \\
\text { method of } \\
\text { preventing } \\
\text { pregnancy } \\
\text { complications } \\
\text { too. }\end{array}$ \\
\hline $\begin{array}{l}\text { Castro et } \\
\text { al. }{ }^{9}\end{array}$ & $\begin{array}{l}\text { Motivational } \\
\text { interviewing } \\
\text { grounded } \\
\text { in social } \\
\text { cognitive } \\
\text { theory }\end{array}$ & Interventional & $\begin{array}{l}400 \\
\text { obese/ } \\
\text { overweight } \\
\text { Latino } \\
\text { smokers }\end{array}$ & $\begin{array}{l}\text { Identified common } \\
\text { mechanisms underlying } \\
\text { change in smoking, } \\
\text { physical activity, and diet } \\
\text { used as treatment targets; } \\
\text { identified practical models } \\
\text { of patient-centered cross- } \\
\text { cultural service provision }\end{array}$ & $\begin{array}{l}\text { A practical } \\
\text { example of how } \\
\text { an intervention } \\
\text { can be adapted } \\
\text { to maximize } \\
\text { relevance and } \\
\text { acceptability and } \\
\text { also maintain the } \\
\text { core elements } \\
\text { of the original } \\
\text { evidence-based } \\
\text { intervention }\end{array}$ & $\begin{array}{l}\text { The } \\
\text { intervention } \\
\text { package was } \\
\text { adopted from } \\
\text { smoking } \\
\text { cessation } \\
\text { program. }\end{array}$ \\
\hline
\end{tabular}




\begin{tabular}{|c|c|c|c|c|c|c|}
\hline $\begin{array}{l}\text { Dewar et } \\
\text { al. }{ }^{10}\end{array}$ & $\begin{array}{l}\text { Intervention, } \\
\text { guided } \\
\text { by Social } \\
\text { Cognitive } \\
\text { Theory. }\end{array}$ & $\begin{array}{l}\text { Group } \\
\text { randomized } \\
\text { controlled } \\
\text { trial with } \\
\text { 12-month } \\
\text { follow-up }\end{array}$ & $\begin{array}{l}357 \\
\text { adolescent } \\
\text { girls }\end{array}$ & $\begin{array}{l}\text { Significant between group } \\
\text { differences in favor of } \\
\text { the intervention group for } \\
\text { self-reported recreational } \\
\text { computer use (-26.0 min; } \\
95 \% \text { CI, }-46.9 \text { to }-5.1) \text {, } \\
\text { and sedentary activities } \\
\text { summed (-56.4 min; } 95 \% \\
\text { CI, }-110.1 \text { to }-2.7) \text {, however } \\
\text { objective sedentary } \\
\text { behavior showed no } \\
\text { differences }\end{array}$ & $\begin{array}{l}\text { Intervention for } \\
\text { adolescent girls } \\
\text { in low-income } \\
\text { communities } \\
\text { significantly } \\
\text { reduced time } \\
\text { spent in sedentary } \\
\text { activities. } \\
\text { However, } \\
\text { improvements in } \\
\text { physical activity } \\
\text { and hypothesized } \\
\text { mediators of } \\
\text { physical activity } \\
\text { behavior were not } \\
\text { observed }\end{array}$ & $\begin{array}{l}\text { Intervention } \\
\text { included } \\
\text { enhanced } \\
\text { school sport, } \\
\text { lunchtime } \\
\text { physical } \\
\text { activity } \\
\text { sessions, } \\
\text { interactive } \\
\text { seminars, } \\
\text { nutrition } \\
\text { workshops, } \\
\text { to encourage } \\
\text { physical } \\
\text { activity and } \\
\text { healthy eating, } \\
\text { and a decrease } \\
\text { in sedentary } \\
\text { behavior. }\end{array}$ \\
\hline $\begin{array}{l}\text { Dewar et } \\
\text { al. }{ }^{11}\end{array}$ & $\begin{array}{l}\text { Multi } \\
\text { component } \\
\text { intervention } \\
\text { guided } \\
\text { by social } \\
\text { cognitive } \\
\text { theory }\end{array}$ & $\begin{array}{l}\text { RCT, the } \\
\text { Nutrition and } \\
\text { Enjoyable } \\
\text { Activity for } \\
\text { Teen Girls } \\
\text { (NEAT Girls) } \\
\text { intervention. }\end{array}$ & $\begin{array}{l}357 \\
\text { adolescent } \\
\text { girls }\end{array}$ & $\begin{array}{l}\text { No intervention effects } \\
\text { on BMI (adjusted mean } \\
\text { difference }-0.33,95 \% \\
\mathrm{CI}=-0.97,0.28, \mathrm{p}=0.353) \\
\text { and BMI z-score }(-0.12, \\
95 \% \mathrm{CI}=-0.27,0.04, \\
\mathrm{p}=0.178) . \text { However, there } \\
\text { was a group-by-time } \\
\text { interaction for percentage } \\
\text { body fat }(-1.96 \%, 95 \% \\
\mathrm{CI}=-3.02,-0.89, \mathrm{p}=0.006) \text {. } \\
\text { Intervention effects for } \\
\text { physical activity, screen } \\
\text { time, and dietary intake } \\
\text { were not significant }\end{array}$ & $\begin{array}{l}\text { The NEAT Girls } \\
\text { intervention } \\
\text { did not result in } \\
\text { effects on BMI }\end{array}$ & $\begin{array}{l}\text { Study of } \\
\text { youth who } \\
\text { are "at risk" } \\
\text { of obesity } \\
\text { should focus } \\
\text { on strategies } \\
\text { to improve } \\
\text { retention and } \\
\text { adherence in } \\
\text { prevention } \\
\text { programs. }\end{array}$ \\
\hline $\begin{array}{l}\text { Puma et } \\
\text { al. }{ }^{12}\end{array}$ & $\begin{array}{l}\text { social } \\
\text { cognitive } \\
\text { theory }\end{array}$ & $\begin{array}{l}\text { Quasi- } \\
\text { experimental } \\
\text { design } \\
\text { comparing } \\
\text { intervention } \\
\text { and } \\
\text { comparison } \\
\text { cohorts }\end{array}$ & 15,000 & $\begin{array}{l}\text { Long-term effects were } \\
\text { observed in nutrition- } \\
\text { related knowledge and } \\
\text { attitudes but not self- } \\
\text { efficacy or behavior change }\end{array}$ & $\begin{array}{l}\text { It Had limited } \\
\text { lasting effects } \\
\text { when students } \\
\text { have increased } \\
\text { autonomy to } \\
\text { make food } \\
\text { choices. }\end{array}$ & $\begin{array}{l}\text { Intervention } \\
\text { can only be } \\
\text { useful when } \\
\text { subjects are } \\
\text { given to } \\
\text { autonomy to } \\
\text { food choices. }\end{array}$ \\
\hline $\begin{array}{l}\text { Rosario } \\
\text { et al. }{ }^{13}\end{array}$ & $\begin{array}{l}\text { Health } \\
\text { Promotion } \\
\text { Model } \\
\text { and social } \\
\text { cognitive } \\
\text { theory. }\end{array}$ & $\begin{array}{l}\text { Randomized } \\
\text { trial }\end{array}$ & $\begin{array}{l}464 \\
\text { subjects }\end{array}$ & $\begin{array}{l}\text { Children from intervention } \\
\text { group reported a reduction } \\
\text { whereas the control group } \\
\text { reported an increase } \\
\text { in solid LNED foods } \\
\text { consumption. The odds } \\
\text { of increasing solid LNED } \\
\text { foods consumption was } \\
0.48,95 \% \mathrm{CI}(0.24,0.95) \text { in } \\
\text { the intervention schools. }\end{array}$ & $\begin{array}{l}\text { Study provides } \\
\text { further support } \\
\text { for the success } \\
\text { of intervention } \\
\text { programs aimed } \\
\text { at limiting the } \\
\text { consumption } \\
\text { of solid LNED } \\
\text { foods in children. }\end{array}$ & $\begin{array}{l}\text { Intervention } \\
\text { program } \\
\text { were held } \\
\text { by teachers } \\
\text { previously } \\
\text { trained in } \\
\text { nutrition, } \\
\text { on the } \\
\text { consumption } \\
\text { of low } \\
\text { nutrient, } \\
\text { energy-dense } \\
\text { (LNED) } \\
\text { foods, of } \\
\text { children } \\
\text { attending } \\
\text { elementary } \\
\text { schools }\end{array}$ \\
\hline
\end{tabular}




\begin{tabular}{|c|c|c|c|c|c|c|}
\hline $\begin{array}{l}\text { Safdie et } \\
\text { al. }^{14}\end{array}$ & $\begin{array}{l}\text { Integration } \\
\text { of ecological } \\
\text { principles } \\
\text { and Social } \\
\text { Cognitive } \\
\text { Theory } \\
\text { (SCT) } \\
\text { constructs }\end{array}$ & $\begin{array}{l}\text { School-based } \\
\text { obesity } \\
\text { prevention } \\
\text { program } \\
\text { implemented } \\
\text { in Mexico }\end{array}$ & $\begin{array}{l}15 \\
\text { schools }\end{array}$ & $\begin{array}{l}\text { The most frequently used } \\
\text { SCT construct within both } \\
\text { intervention domains was } \\
\text { Reciprocal Determinism } \\
\text { (e.g., where changes to } \\
\text { the environment influence } \\
\text { changes in behavior and } \\
\text { these behavioral changes } \\
\text { influence further changes } \\
\text { to the environment); no } \\
\text { significant differences were } \\
\text { observed in the use of SCT } \\
\text { constructs across domains. }\end{array}$ & $\begin{array}{l}\text { Promising } \\
\text { combination } \\
\text { of strategies } \\
\text { and theoretical } \\
\text { constructs that } \\
\text { can be used } \\
\text { to implement } \\
\text { a school- } \\
\text { based obesity } \\
\text { prevention } \\
\text { program }\end{array}$ & $\begin{array}{l}\text { Strategies } \\
\text { emphasized } \\
\text { school-level } \\
\text { infrastructure/ } \\
\text { personnel } \\
\text { change } \\
\text { and strong } \\
\text { political } \\
\text { engagement } \\
\text { and were most } \\
\text { commonly } \\
\text { underpinned } \\
\text { by Reciprocal } \\
\text { Determinism } \\
\text { for both } \\
\text { Nutrition } \\
\text { and Physical } \\
\text { Activity. }\end{array}$ \\
\hline $\begin{array}{l}\text { Smith et } \\
\text { al. }{ }^{15}\end{array}$ & $\begin{array}{l}\text { Self- } \\
\text { determination } \\
\text { theory } \\
\text { and social } \\
\text { cognitive } \\
\text { theory. }\end{array}$ & $\begin{array}{l}\text { 'Active Teen } \\
\text { Leaders } \\
\text { Avoiding } \\
\text { Screen-time' } \\
\text { (ATLAS) } \\
\text { obesity } \\
\text { prevention } \\
\text { intervention } \\
\text { in low- } \\
\text { income } \\
\text { communities }\end{array}$ & 22 & $\begin{array}{l}\text { Primary outcomes } \\
\text { were (BMI) and waist } \\
\text { circumference. Secondary } \\
\text { outcomes include BMI } \\
\text { z-scores, body fat muscular } \\
\text { fitness (grip strength and } \\
\text { push-ups), screen-time, } \\
\text { sugar-sweetened beverage } \\
\text { consumption, resistance } \\
\text { training skill competency, } \\
\text { daytime sleepiness, } \\
\text { subjective well-being, } \\
\text { physical self-perception, } \\
\text { pathological video gaming, } \\
\text { and aggression. }\end{array}$ & $\begin{array}{l}\text { ATLAS is an } \\
\text { innovative } \\
\text { school-based } \\
\text { intervention } \\
\text { designed to } \\
\text { improve the } \\
\text { health behaviors } \\
\text { and related } \\
\text { outcomes of } \\
\text { adolescent males } \\
\text { in low-income } \\
\text { communities. }\end{array}$ & $\begin{array}{l}\text { ATLAS can } \\
\text { be used as } \\
\text { an effective } \\
\text { intervention } \\
\text { for obesity } \\
\text { prevention in } \\
\text { developing } \\
\text { countries as } \\
\text { well. }\end{array}$ \\
\hline $\begin{array}{l}\text { Smith et } \\
\text { al. }{ }^{16}\end{array}$ & $\begin{array}{l}\text { Guided } \\
\text { by self- } \\
\text { determination } \\
\text { theory } \\
\text { and social } \\
\text { cognitive } \\
\text { theory. }\end{array}$ & $\begin{array}{l}\text { Cluster } \\
\text { randomized } \\
\text { controlled } \\
\text { trial } \\
\text { conducted in } \\
14 \text { secondary } \\
\text { schools in } \\
\text { low-income } \\
\text { communities }\end{array}$ & 361 & $\begin{array}{l}\text { No significant intervention } \\
\text { effects for BMI, waist } \\
\text { circumference, percent } \\
\text { body fat, or physical } \\
\text { activity. Significant } \\
\text { intervention effects found } \\
\text { for screen-time (mean }+/- \\
\mathrm{SE} \text { : }-30+/-10.08 \text { min } / \mathrm{d} \text {; } \\
\mathrm{P}=.03 \text { ), sugar-sweetened } \\
\text { beverage consumption } \\
\text { (mean: }-0.6 \pm 0.26 \\
\text { glass } / \mathrm{d} ; \mathrm{P}=.01 \text { ), muscular } \\
\text { fitness (mean: } 0.9 \pm 0.49 \\
\text { repetition; } \mathrm{P}=.04 \text { ), and } \\
\text { resistance training skills } \\
\text { (mean: } 5.7+/-0.67 \text { units; } \mathrm{P} \\
<.001 \text { ). }\end{array}$ & $\begin{array}{l}\text { School-based } \\
\text { intervention } \\
\text { targeting } \\
\text { low-income } \\
\text { adolescent boys } \\
\text { did not result } \\
\text { in significant } \\
\text { effects on body } \\
\text { composition, } \\
\text { perhaps due to } \\
\text { an insufficient } \\
\text { activity dose. }\end{array}$ & $\begin{array}{l}\text { Intervention } \\
\text { was successful } \\
\text { in improving } \\
\text { muscular } \\
\text { fitness, } \\
\text { movement } \\
\text { skills, and key } \\
\text { weight-related } \\
\text { behaviors. }\end{array}$ \\
\hline
\end{tabular}




\begin{tabular}{|c|c|c|c|c|c|c|}
\hline $\begin{array}{l}\text { Leach et } \\
\text { al. }{ }^{17}\end{array}$ & $\begin{array}{l}\text { Baseline } \\
\text { nutrition } \\
\text { intervention, } \\
\text { grounded } \\
\text { in Social } \\
\text { Cognitive } \\
\text { Theory }\end{array}$ & $\begin{array}{l}\text { Intervention } \\
\text { on blinded } \\
\text { cohorts used } \\
\text { on study } \\
\text { participant in } \\
\text { Mississippi } \\
\text { public school } \\
\text { students }\end{array}$ & $\begin{array}{l}22 \\
\text { volunteers } \\
\text { of } 57 \\
\text { eligible, } \\
\text { overweight } \\
\text { female }\end{array}$ & $\begin{array}{l}\text { No pre intervention } \\
\text { differences were found } \\
\text { in height, weight, BMI, } \\
\text { or age. Higher follow-up } \\
\text { BMI scores were found in } \\
\text { both groups, and. Gains } \\
\text { in nutrition knowledge } \\
\text { were sustained }(\mathrm{P}<.002) \text {; } \\
\text { however, there was no } \\
\text { association between } \\
\text { nutrition knowledge and } \\
\text { follow-up BMI }(\mathrm{r}=-.185 ; \mathrm{P} \\
<.462) \text {. }\end{array}$ & $\begin{array}{l}\text { Minimal nutrition } \\
\text { education } \\
\text { alone may be } \\
\text { an ineffective } \\
\text { intervention } \\
\text { for overweight } \\
\text { children. Provides } \\
\text { an example of } \\
\text { how youth soccer } \\
\text { may benefit } \\
\text { overweight } \\
\text { children }\end{array}$ & $\begin{array}{l}\text { No significant } \\
\text { differences } \\
\text { between } \\
\text { groups } \\
\text { were found, } \\
\text { possibly } \\
\text { because of } \\
\text { the small } \\
\text { sample sizes } \\
\text { and the short } \\
\text { 8-week soccer } \\
\text { intervention } \\
\text { period }\end{array}$ \\
\hline $\begin{array}{l}\text { Lubans } \\
\text { et al. }{ }^{18}\end{array}$ & $\begin{array}{l}\text { Social } \\
\text { Cognitive } \\
\text { Theory } \\
\text { (SCT) } \\
\text { assessed } \\
\text { using a } \\
\text { questionnaire. }\end{array}$ & $\begin{array}{l}\text { Group } \\
\text { randomized } \\
\text { controlled } \\
\text { trial on One } \\
\text { hundred } \\
\text { adolescent } \\
\text { boys (mean } \\
\text { age }=14.3 \\
(0.6) \text { years) }\end{array}$ & 100 & $\begin{array}{l}\text { Intervention had significant } \\
\text { effect on resistance training } \\
\text { self-efficacy }(\mathrm{p}<0.001) \text {, but } \\
\text { none of the } \mathrm{SCT} \text { constructs } \\
\text { satisfied the criteria for } \\
\text { mediation. Changes in } \\
\text { BMI were also associated } \\
\text { with changes in resistance } \\
\text { training self-efficacy } \\
(\mathrm{r}=-0.21, \mathrm{p}=0.06) \text { and } \\
\text { physical activity behavioral } \\
\text { strategies }(\mathrm{r}=-0.29, \\
\mathrm{p}=0.009) .\end{array}$ & $\begin{array}{l}\text { Intervention } \\
\text { incorporating } \\
\text { student leadership } \\
\text { increased } \\
\text { adolescent boys' } \\
\text { resistance training } \\
\text { self-efficacy, } \\
\text { but changes in } \\
\text { physical activity } \\
\text { were not detected } \\
\text { and none of the } \\
\text { SCT constructs } \\
\text { satisfied the } \\
\text { criteria for } \\
\text { mediation. }\end{array}$ & $\begin{array}{l}\text { Baseline } \\
\text { weight } \\
\text { status was a } \\
\text { moderator of } \\
\text { intervention } \\
\text { effect with } \\
\text { the strongest } \\
\text { intervention } \\
\text { effects } \\
\text { observed } \\
\text { among } \\
\text { overweight } \\
\text { and obese } \\
\text { adolescent } \\
\text { boys. }\end{array}$ \\
\hline $\begin{array}{l}\text { Mastin } \\
\text { et al. }{ }^{19}\end{array}$ & $\begin{array}{l}\text { Social } \\
\text { cognitive } \\
\text { theory } \\
\text { framework }\end{array}$ & Observational & $\begin{array}{l}46 \\
\text { women }\end{array}$ & $\begin{array}{l}\text { Although participants' } \\
\text { primary weight- } \\
\text { related obstacles were } \\
\text { environment-based, } \\
\text { for example, unsafe } \\
\text { environments in which to } \\
\text { engage in regular exercise, } \\
\text { they more often offered } \\
\text { individual-based solutions. }\end{array}$ & $\begin{array}{l}\text { Discussion of } \\
\text { media advocacy } \\
\text { as a tool that } \\
\text { can be used } \\
\text { to promote } \\
\text { environmental } \\
\text { solutions. }\end{array}$ & $\begin{array}{l}\text { Media } \\
\text { advocacy } \\
\text { can be used } \\
\text { to increase } \\
\text { effectiveness } \\
\text { of } \\
\text { interventions. }\end{array}$ \\
\hline $\begin{array}{l}\text { Mead et } \\
\text { al. }{ }^{20}\end{array}$ & $\begin{array}{l}\text { Social } \\
\text { cognitive } \\
\text { theory } \\
\text { and Social } \\
\text { ecological } \\
\text { models }\end{array}$ & $\begin{array}{l}\text { Community- } \\
\text { based, } \\
\text { multi-level } \\
\text { intervention } \\
\text { using } \\
\text { formative } \\
\text { research and } \\
\text { a community } \\
\text { participatory } \\
\text { process. }\end{array}$ & $\begin{array}{l}246 \\
\text { adults }\end{array}$ & $\begin{array}{l}\text { Respondents living in } \\
\text { intervention communities } \\
\text { showed significant } \\
\text { improvements in food- } \\
\text { related self-efficacy (beta } \\
=0.15, \mathrm{p}=.003 \text { ) and } \\
\text { intentions (beta }=0.16 \text {, } \\
\mathrm{p}=.001 \text { ) compared with } \\
\text { comparison communities. }\end{array}$ & $\begin{array}{l}\text { More } \\
\text { improvements } \\
\text { from the } \\
\text { intervention } \\
\text { were seen in } \\
\text { overweight, } \\
\text { obese, and high } \\
\text { socioeconomic } \\
\text { status } \\
\text { respondents. }\end{array}$ & $\begin{array}{l}\text { Community- } \\
\text { based, } \\
\text { multilevel } \\
\text { intervention } \\
\text { is an effective } \\
\text { strategy to } \\
\text { improve } \\
\text { psychosocial } \\
\text { factors for } \\
\text { healthy } \\
\text { nutritional } \\
\text { behavior } \\
\text { change to } \\
\text { reduce chronic } \\
\text { disease in } \\
\text { indigenous } \\
\text { populations }\end{array}$ \\
\hline
\end{tabular}




\begin{tabular}{|c|c|c|c|c|c|c|}
\hline $\begin{array}{l}\text { Winett et } \\
\text { al. }^{21}\end{array}$ & $\begin{array}{l}\text { Social } \\
\text { cognitive } \\
\text { theory (SCT) }\end{array}$ & $\begin{array}{l}\text { Internet-based } \\
\text { intervention }\end{array}$ & - & $\begin{array}{l}\text { The high use of the Internet } \\
\text { provides a vehicle to } \\
\text { reach different population } \\
\text { segments with readily } \\
\text { accessible, SCT-tailored } \\
\text { long-term programs. } \\
\text { Research studies using } \\
\text { the Internet with tailored } \\
\text { SCT interventions have } \\
\text { shown changes in nutrition } \\
\text { practices, physical activity, } \\
\text { and weight loss for up to a } \\
\text { year }\end{array}$ & $\begin{array}{l}\text { One promising } \\
\text { approach to weight } \\
\text { gain prevention } \\
\text { in population } \\
\text { segments is the } \\
\text { development and } \\
\text { wide spread use } \\
\text { of longer-term } \\
\text { Internet programs } \\
\text { using specific } \\
\text { principles and } \\
\text { procedures from } \\
\text { SCT. }\end{array}$ & $\begin{array}{l}\text { More dynamic } \\
\text { use of social } \\
\text { cognitive } \\
\text { theory (SCT) } \\
\text { for developing } \\
\text { programs } \\
\text { to maintain } \\
\text { health behavior } \\
\text { changes is } \\
\text { emerging with } \\
\text { some evidence } \\
\text { of long-term } \\
\text { maintenance }\end{array}$ \\
\hline Li et al. ${ }^{22}$ & $\begin{array}{l}\text { Social } \\
\text { cognitive } \\
\text { theory }\end{array}$ & $\begin{array}{l}\text { Cross- } \\
\text { sectional } \\
\text { survey }\end{array}$ & $\begin{array}{l}2400 \\
\text { children }\end{array}$ & $\begin{array}{l}15.2 \% \text { of children were } \\
\text { overweight and } 10.9 \% \\
\text { were obese; nearly } 80 \% \\
\text { of children spent inverted } \\
\text { question mark } 2 \text { hrs./day } \\
\text { either on physical activities } \\
\text { or screen time. }\end{array}$ & $\begin{array}{l}\text { Screen time is } \\
\text { independently } \\
\text { associated with } \\
\text { childhood obesity, } \\
\text { and needs be } \\
\text { focused for } \\
\text { obesity prevention } \\
\text { in school-aged } \\
\text { children in China. }\end{array}$ & $\begin{array}{l}\text { Compared with } \\
\text { those spent } \\
>3 \text { hrs./day on } \\
\text { screen time, } \\
\text { children who } \\
\text { spent inverted } \\
\text { question mark2 } \\
\text { hrs./day or } \\
\text { between 2-3 } \\
\text { hrs./day were } \\
\text { significantly } \\
\text { less likely to } \\
\text { be obese after } \\
\text { adjusting for } \\
\text { other variables }\end{array}$ \\
\hline $\begin{array}{l}\text { Neumark- } \\
\text { Sztainer } \\
\text { et al. }\end{array}$ & $\begin{array}{l}\text { Social } \\
\text { cognitive } \\
\text { theory }\end{array}$ & $\begin{array}{l}\text { Cohort study } \\
\text { performed }\end{array}$ & 201 girls & $\begin{array}{l}\text { The two strongest and most } \\
\text { consistent factors associated } \\
\text { with change in physical } \\
\text { activity were time constraints } \\
\text { and support for physical } \\
\text { activity from peers, parents, } \\
\text { and teachers }\end{array}$ & $\begin{array}{l}\text { Effectiveness } \\
\text { of interventions } \\
\text { aimed at } \\
\text { increasing } \\
\text { physical activity } \\
\text { among adolescent } \\
\text { girls might be } \\
\text { enhanced by } \\
\text { engaging support } \\
\text { from friends, } \\
\text { family, and caring } \\
\text { adults which may } \\
\text { help in obesity } \\
\text { prevention }\end{array}$ & $\begin{array}{l}\text { Physical } \\
\text { activity is } \\
\text { an important } \\
\text { component } \\
\text { of a healthy } \\
\text { lifestyle, with } \\
\text { implications } \\
\text { for the } \\
\text { prevention } \\
\text { of chronic } \\
\text { diseases and } \\
\text { obesity. }\end{array}$ \\
\hline $\begin{array}{l}\text { Arikan et } \\
\text { al. }^{24}\end{array}$ & $\begin{array}{l}\text { Social } \\
\text { cognitive } \\
\text { Theory }\end{array}$ & $\begin{array}{l}\text { Randomized } \\
\text { control trial }\end{array}$ & $\begin{array}{l}2,038 \\
\text { subjects }\end{array}$ & $\begin{array}{l}\text { Of the total participants, } \\
85.2 \% \text { learned about } \\
\text { the "Fighting-Obesity } \\
\text { Campaign" through } \\
\text { television, } 28.1 \% \text { through } \\
\text { radio, } 11.0 \% \text { from } \\
\text { newspapers, } 6.0 \% \text { from } \\
\text { billboards, and } 19.2 \% \text { from } \\
\text { other sources. Study revealed } \\
\text { that } 28.5 \% \text { of the participants } \\
\text { adopted desired behavioral } \\
\text { changes after exposure } \\
\text { to campaign. Logistic } \\
\text { regression demonstrated that } \\
\text { behavior change is greater on } \\
\text { women. }\end{array}$ & $\begin{array}{l}\text { Media campaigns } \\
\text { may cause } \\
\text { behavioral changes } \\
\text { by increasing } \\
\text { motivation to } \\
\text { prevent obesity } \\
\text { within the target } \\
\text { population. }\end{array}$ & $\begin{array}{l}\text { Continuing } \\
\text { these types of } \\
\text { campaigns can } \\
\text { lead to success } \\
\text { at the national } \\
\text { level too. }\end{array}$ \\
\hline
\end{tabular}




\section{DISCUSSION}

Types of obesity prevention interventions targeting social relational constructs and characterized by the degree to which these interventions have addressed key social relational constructs in intervention design and implementation play importantly in overweight and obesity. Social cognitive theory does not propose that increasing self-efficacy will inevitably result in behavior change ${ }^{25}$. The theory states that the effects of self-efficacy on behavior will be moderated by outcome expectancies, i.e. beliefs that a particular behavior will lead to a particular outcome. Where an individual believes that the behavior will not lead to a valued outcome, self-efficacy will not motivate behavior change. For example, an individual may believe they can drink fewer alcoholic drinks, but if they do not think the amount they are drinking is harmful, such self-efficacy will not result in less consumption. In terms of the present review, obese individuals do not believe that increasing their physical activity will lead to weight loss, which presumably would be a highly valued goal. There is evidence that the relationship between increased physical activity and weight loss is far from straightforward ${ }^{26}$, so that this would be reasonable outcome expectancy for many obese people. Thus, this population may be convinced by an intervention that they can increase their physical activity, but if they were not convinced that this would result in the salient outcome of weight loss, it would not necessarily result in increased physical activity. Apart from this only the construct "self - efficacy" of social cognitive theory has been widely used in many of its interventions. ${ }^{27}$ In this study media campaign was launched grounded in social cognitive theory logistic regression results demonstrated that behavior change is greater among women. ${ }^{24}$ There were a range of outcomes found in the set of interventions. Obesity-related outcomes included (1) anthropometric indicators, such as body mass index or body fat percentage, (2) physiological measures of cholesterol, blood pressure, and blood sugar, and (3) behavioral risk factors such as physical activity, dietary patterns and knowledge, screen time, sedentary time, and smoking. Anumber of studies included psychological and psychosocial outcomes, such as depressive symptoms, self- efficacy, and motivation, while some studies also included social indicators, such as social support. Furthermore, the frequent reference to self-efficacy in the selected interventions requires additional attention. Self-efficacy; which comprises an individual's motivation, locus of control, behavioral choices, intentions, and actions with respect to their goals, tasks, and challenges; was often included as a predictor, mediator, or moderator of overweight and obesity risk factors and status. The theoretical emphasis on personal responsibility and control belies the use of concepts related to social, political, and organizational change. ${ }^{28}$ This is not to detract from the value of individually oriented theories. ${ }^{29}$ However, mounting evidence suggests that innovative strategies for addressing and preventing obesity at a population level should entail theories and approaches that operate from an ecological perspective. ${ }^{30}$ Integrative research review applying Stetler's model of research utilization also revealed that constructs of social cognitive/learning theory are effective in eight out of ten studies to prevent and treat childhood obesity among four to 14 years children. ${ }^{31}$

\section{CONCLUSION}

Self-efficacy and social support are the important constructs of social cognitive theory in obesity or overweight prevention or maintaining normal body weight. Other constructs remained to be modest even among the children. Media effects and ecological components add the effectiveness.

\section{RECOMMENDATIONS}

It is recommended that ecological components like media campaign or internet should be integrated with any of the constructs such as self-efficacy and social supports, when applying social cognitive theory to prevent obesity or overweight. Internet is becoming even a more useful tool in such.

\section{AUTHORS' CONTRIBUTIONS}

CA developed the review guideline and oversaw all aspects of this study. AP, DT, RT, SM and SG retrieved the articles; extracted data; summarized findings; and prepared the draft manuscript. AP reviewed and provided the valuable feedback. All authors provided valuable contributions to the 
development and refinement of the manuscript including intellectual content. CA reviewed and incorporated the feedback and prepared the final manuscript. All authors read and provided approval of the completed manuscript.

\section{ACKNOWLEDGEMENTS}

Authors duly acknowledge to all the authors of retrieved original articles and surveys.

\section{CONFLICTS OF INTEREST}

The authors declare that they have no competing interests.

\section{ETHICAL APPROVAL AND CONSENT TO PARTICIPATE}

Not applicable.

\section{AVAILABILITY OF DATA AND MATERIALS}

Not applicable.

\section{REFERENCES}

1. Godin G, Amireault S, Belanger-Gravel A, Vohl MC, Perusse L. Prediction of leisure-time physical activity among obese individuals. Obesity (Silver Spring). 2009 Apr;17(4):70612

2. Kopelman PG. Obesity as a medical problem. Nature. 2000;404(6778):635-43.

3. Gray-Donald K, Robinson E, Collier A, David K, Renaud L, Rodrigues S. Intervening to reduce weight gain in pregnancy and gestational diabetes mellitus in Cree communities: an evaluation. CMAJ. 2000 Nov 14;163(10):1247-51.

4. Dewar DL, Plotnikoff RC, Morgan PJ, Okely AD, Costigan SA, Lubans DR. Testing socialcognitive theory to explain physical activity change in adolescent girls from low-income communities. Res Q Exerc Sport. 2013 Dec;84(4):483-91.

5. Dressler H, Smith C. Environmental, personal, and behavioral factors are related to body mass index in a group of multi-ethnic, lowincome women. J Acad Nutr Diet. 2013 Dec;113(12):1662-8.

6. Patrick K, Marshall SJ, Davila EP, Kolodziejczyk JK, Fowler JH, Calfas KJ, et al.
Design and implementation of a randomized controlled social and mobile weight loss trial for young adults (project SMART). Contemp Clin Trials. 2014 Jan;37(1):10-8.

7. Sharpe PA, Burroughs EL, Granner ML, Wilcox S, Hutto BE, Bryant CA, et al. Impact of a community-based prevention marketing intervention to promote physical activity among middle-aged women. Health Educ Behav. 2010 Jun;37(3):403-23.

8. Briley AL, Barr S, Badger S, Bell R, Croker H, Godfrey KM, et al. A complex intervention to improve pregnancy outcome in obese women; the UPBEAT randomised controlled trial. BMC Pregnancy Childbirth. 2014;14:74.

9. Castro Y, Fernandez ME, Strong LL, Stewart DW, Krasny S, Hernandez Robles E, et al. Adaptation of a counseling intervention to address multiple cancer risk factors among overweight/obese latino smokers. Health Educ Behav. 2015 Feb;42(1):65-72.

10. Dewar DL, Morgan PJ, Plotnikoff RC, Okely AD, Batterham M, Lubans DR. Exploring changes in physical activity, sedentary behaviors and hypothesized mediators in the NEAT girls group randomized controlled trial. J Sci Med Sport. 2014 Jan;17(1):39-46.

11. Dewar DL, Morgan PJ, Plotnikoff RC, Okely $\mathrm{AD}$, Collins $\mathrm{CE}$, Batterham $\mathrm{M}$, et al. The nutrition and enjoyable activity for teen girls study: a cluster randomized controlled trial. Am J Prev Med. 2013 Sep;45(3):313-7.

12. Puma J, Romaniello C, Crane L, Scarbro S, Belansky E, Marshall JA. Long-term student outcomes of the Integrated Nutrition and Physical Activity Program. J Nutr Educ Behav. 2013 Nov-Dec;45(6):635-42.

13. Rosario R, Araujo A, Oliveira B, Padrao $\mathrm{P}$, Lopes $\mathrm{O}$, Teixeira $\mathrm{V}$, et al. Impact of an intervention through teachers to prevent consumption of low nutrition, energy-dense foods and beverages: a randomized trial. Prev Med. 2013 Jul;57(1):20-5.

14. Safdie M, Cargo M, Richard L, Levesque L. An ecological and theoretical deconstruction of a school-based obesity prevention program in Mexico. Int J Behav Nutr Phys Act. 2014 Aug 10;11(1):103.

15. Smith JJ, Morgan PJ, Plotnikoff RC, Dally KA, 
Salmon J, Okely AD, et al. Rationale and study protocol for the 'active teen leaders avoiding screen-time' (ATLAS) group randomized controlled trial: an obesity prevention intervention for adolescent boys from schools in low-income communities. Contemp Clin Trials. 2014 Jan;37(1):106-19.

16. Smith JJ, Morgan PJ, Plotnikoff RC, Dally KA, Salmon J, Okely AD, et al. Smart-phone obesity prevention trial for adolescent boys in low-income communities: the ATLAS RCT. Pediatrics. 2014 Sep;134(3):e723-31.

17. Leach RA, Yates JM. Nutrition and youth soccer for childhood overweight: a pilot novel chiropractic health education intervention. J Manipulative Physiol Ther. 2008 JulAug;31(6):434-41.

18. Lubans DR, Morgan PJ, Callister R. Potential moderators and mediators of intervention effects in an obesity prevention program for adolescent boys from disadvantaged schools. J Sci Med Sport. 2012 Nov;15(6):519-25.

19. Mastin T, Campo S, Askelson NM. African American women and weight loss: disregarding environmental challenges. J Transcult Nurs. 2012 Jan;23(1):38-45.

20. Mead EL, Gittelsohn J, Roache C, Corriveau A, Sharma S. A community-based, environmental chronic disease prevention intervention to improve healthy eating psychosocial factors and behaviors in indigenous populations in the Canadian Arctic. Health Educ Behav. 2013 Oct;40(5):592-602.

21. Winett RA, Tate DF, Anderson ES, Wojcik JR, Winett SG. Long-term weight gain prevention: a theoretically based Internet approach. Prev Med. 2005 Aug;41(2):629-41.

22. Li L, Shen T, Wen L, Wu M, He P, Wang Y, et al. Lifestyle factors associated with childhood obesity: a cross-sectional study in Shanghai, China. BMC Res Notes. 2015 Jan 17;8(1):6.

23. Neumark-Sztainer D, Story M, Hannan PJ, Tharp T, Rex J. Factors associated with changes in physical activity: a cohort study of inactive adolescent girls. Archives of Pediatrics \& Adolescent Medicine. 2003;157(8):803-10.

24. Arikan I, Karakaya K, Erata M, Tuzun H, Baran E, Levent G, et al. Fighting obesity campaign in Turkey: evaluation of media campaign efficacy. Cent Eur J Public Health. 2014 Sep;22(3):170-4.

25. Rosenthal R: Meta-analytic procedures for social research. Beverly Hills, CA: Sage; 1984.

26. Ebersole K, Dugas L, Durazo-Arvizu RA, Adeyemo AA, Tayo BO, Omotade OO, Brieger W, Schoeller DA, Cooper RS, Luke A: Energy expenditure and adiposity in Nigerian and African American women. Obesity 2008, 16:2148-2154.

27. Tan, E.J., Rebok, G.W., Frangakis, C.E., Carlson, M.C., Wang, T., Ricks, M., Tanner, E.K., McGill, S., Fried, L.P. The longterm relationship between high-intensity volunteering and physical activity in older African American women. Journal of Gerontology Series B, 64B(2), 2009, p 304.

28. T. A. Glass and M. J. McAtee, "Behavioral science at the crossroads in public health: extending horizons, envisioning the future," Social Science and Medicine, vol. 62, no. 7, pp. 1650-1671, 2006.

29. K. Glanz and D. B. Bishop, "The role of behavioral science theory in development and implementation of public health interventions," Annual Review of Public Health, vol. 31, pp. 399-418, 2010.

30. T. T.-K. Huang and T. A. Glass, "Transforming research strategies for understanding and preventing obesity," Journal of the American Medical Association, vol. 300, no. 15, pp. 1811-1813, 2008.

31. Cole K, Waldrop J, D'Auria J, Garner H. An integrative research review: effective schoolbased childhood overweight interventions. J Spec Pediatr Nurs. 2006 Jul;11(3):166-77

32. 